\title{
GROUP RINGS SATISFYING A POLYNOMIAL IDENTITY. II
}

\author{
D. S. PASSMAN
}

Abstract. Let $K[G]$ denote the group ring of $G$ over the field $K$ and let $\Delta$ denote the F.C. subgroup of $G$. In this paper we show that if $K[G]$ satisfies a polynomial identity of degree $n$, then $[G: \Delta] \leqq$ $n / 2$. Moreover this bound is best possible.

If $K[G]$ satisfies a polynomial identity of degree $n$, then it is known that $[G: \Delta]<\infty$. In fact if $K[G]$ is prime or if $K$ has characteristic 0 then $[G: \Delta] \leqq(n / 2)^{2}$ by the results of [4]. In general we have $[G: \Delta] \leqq n !$ by the results of [1]. Thus the goal of this paper is to sharpen these to obtain the best possible bound, namely $[G: \Delta] \leqq n / 2$. We follow the notation of [3].

1. The abelian case. Throughout this section we assume that $[G: \Delta]<\infty$ and that $\Delta$ is abelian. Let $x_{1}=1, x_{2}, x_{3}, \cdots, x_{m}$ be a complete set of $m=[G: \Delta]$ coset representatives for $\Delta$ in $G$.

LEMMA 1.1. There exists a K-monomorphism $\rho: K[G] \rightarrow K[\Delta]_{m}$, where the latter is the ring of $m \times m$ matrices over $K[\Delta]$, satisfying

(i) for $a \in \Delta, \rho(a)=\operatorname{diag}\left(a^{x_{1}}, a^{x_{2}}, \cdots, a^{x_{m}}\right)$,

(ii) $\rho\left(x_{i}\right) e_{11}=e_{i 1}, e_{11} \rho\left(x_{i}^{-1}\right)=e_{1 i}$, where $\left\{e_{i j}\right\}$ is the set of matrix units in $K[\Delta]_{m}$.

Proof. Since $\Delta$ is normal in $G,\left\{x_{1}^{-1}, x_{2}^{-1}, \cdots, x_{m}^{-1}\right\}$ is also a complete set of coset representatives for $\Delta$ in $G$. Set $V=K[G]$. Then clearly $V$ is a left $K[\Delta]$-module with free basis $\left\{x_{1}^{-1}, x_{2}^{-1}, \cdots, x_{m}^{-1}\right\}$. Now $V$ is also a right $K[G]$-module and as such it is faithful. Since right and left multiplication commute as operators on $V$, it follows that $K[G]$ is a set of $K[\Delta]$-linear transformations on an $m$-dimensional free $K[\Delta]$-module $V$. Thus there exists a $K$-monomorphism $\rho$ with $\rho(K[G]) \subseteq K[\Delta]_{m}$.

Let $a \in \Delta$. Then $x_{i}^{-1} a=\left(x_{i}^{-1} a x_{i}\right) x_{i}^{-1}=a^{x_{i}} x_{i}^{-1} ;$ so clearly $\rho(a)=$ $\operatorname{diag}\left(a^{x_{1}}, a^{x_{2}}, \cdots, a^{x_{m}}\right)$.

Now to compute $e_{11} \rho\left(x_{i}^{-1}\right)$ we need only consider the first row of the matrix $\rho\left(x_{i}^{-1}\right)$. Since $x_{1} x_{i}^{-1}=x_{i}^{-1}$ we see that this first row is precisely $e_{1 i}$; so $e_{11} \rho\left(x_{i}^{-1}\right)=e_{11} e_{1 i}=e_{1 i}$.

Received by the editors February 18, 1971 .

AMS 1970 subject classifications. Primary 16A26; Secondary 16A38.

Key words and phrases. Group ring, polynomial identity, F.C. subgroup. 
Finally to compute $\rho\left(x_{i}\right) e_{11}$ we need only look at the first column of the matrix $\rho\left(x_{i}\right)$. Since $x_{j}^{-1} x_{i} \notin \Delta$ for $j \neq i$ and since $x_{j}^{-1} x_{i}=1=x_{1}$ for $j=i$ we see that this first column is precisely $e_{i 1}$. Thus $\rho\left(x_{i}\right) e_{11}=e_{i 1} e_{11}=$ $e_{i 1}$ and the result follows.

Let $K[\Delta]$ be embedded naturally in $K[\Delta]_{m}$ as the set of scalar matrices. Since $\Delta$ is abelian this is a central subring of $K[\Delta]_{m}$. Let $R=K[\Delta]$ - $\rho(K[G])$ be the subring of $K[\Delta]_{m}$ generated by $K[\Delta]$ and $\rho(K[G])$. We will show below that $R$ is in some sense a large subring of $K[\Delta]_{m}$.

LemMA 1.2. For each $i=2,3, \cdots, m$, set

$$
H_{i}=\left\{\left(a, x_{i}\right)=a^{-1} x_{i}^{-1} a x_{i} \mid a \in \Delta\right\} .
$$

Then $H_{i}$ is an infinite subgroup of $\Delta$.

Proof. Since $\Delta$ is a normal abelian subgroup of $G$, the map $\eta_{i}: \Delta \rightarrow \Delta$ given by $a \rightarrow a^{-1} a^{x_{i}}$ is an endomorphism. Clearly $H_{i}$ is the image of $\eta_{i}$ so $H_{i}$ is a subgroup of $\Delta$ and $C_{\Delta}\left(x_{i}\right)$ is the kernel of $\eta_{i}$. Thus $\left[\Delta: C_{\Delta}\left(x_{i}\right)\right]=$ $\left|H_{i}\right|$. If $\left|H_{i}\right|<\infty$, then $\left[\Delta: C_{\Delta}\left(x_{i}\right)\right]<\infty$ and since $[G: \Delta]<\infty$ we would have $\left[G: C_{G}\left(x_{i}\right)\right]<\infty$ and $x_{i} \in \Delta$, a contradiction. Thus $H_{i}$ is infinite.

For each $i=2,3, \cdots, m$, let $S_{i}$ be the augmentation ideal of $K\left[H_{i}\right]$. Thus

$$
S_{i}=\left\{\sum k_{g} g \in K\left[H_{i}\right] \mid \sum k_{g}=0\right\} .
$$

Then $S_{i}$ is a $K$-algebra (without 1 ) which has as a $K$-basis the elements $1-g$ with $g \in H_{i}, g \neq 1$.

Now $S_{i} \subseteq K[\Delta]$ and $K[\Delta]$ is commutative. We define $S=S_{2} S_{3} \cdots S_{m}$ to be the set of all finite $K$-linear sums of products $s_{2} s_{3} \cdots s_{m}$ with $s_{i} \in S_{i}$. Since $K[\Delta]$ is commutative, $S$ is a $K$-subalgebra (without 1 ) of $K[\Delta]$.

LEMMA 1.3. S $\mathrm{S}$ is not a nilpotent ring.

Proof. It clearly suffices to show that for each $i=2,3, \cdots, m$ and $\alpha \in K[\Delta]$ that $S_{i} \alpha=0$ implies $\alpha=0$. Suppose $S_{i} \alpha=0$ and let $g \in H_{i}$. Then $1-g \in S_{i}$ so $(1-g) \alpha=0$. Thus $\alpha=g \alpha$ and $(\operatorname{Supp} \alpha)=g(\operatorname{Supp} \alpha)$. Therefore $H_{i}$ permutes by left multiplication the finite set Supp $\alpha \subseteq \Delta$. If $\alpha \neq 0$ then Supp $\alpha \neq \varnothing$ and this would imply easily that $H_{i}$ is finite, a contradiction by Lemma 1.2. Thus $\alpha=0$ and the result follows.

LEMMA 1.4. With the above notation we have $R \supseteq(S)_{m}$, the ring of $m \times m$ matrices over $S$.

Proof. Recall that $K[\Delta]$ is contained in $K[\Delta]_{m}$ as scalar matrices and that $R=K[\Delta] \cdot \rho(K[G])$. Let $i=2,3, \cdots$, or $m$ and let $a \in \Delta$. Then

$$
a^{-1}\left(\rho(a)-a^{x_{i}}\right) \in R \text {. }
$$


The above matrix is diagonal and we will consider the 1 st and $i$ th entries. The $i$ th entry is $a^{-1}\left(a^{x_{i}}-a^{x_{i}}\right)=0$ by Lemma 1.1(i) and the 1st entry is

$$
a^{-1}\left(a-a^{x_{i}}\right)=1-a^{-1} a^{x_{i}}=1-\left(a, x_{i}\right) \text {. }
$$

Thus for any element $g \in H_{i}, R$ contains a matrix of the form

$$
\operatorname{diag}(1-g, *, 0, *)
$$

where the 0 is in the $i$ th position. Since $R$ is a $K$-algebra and since every element of $S_{i}$ is a $K$-linear sum of such terms $1-g$ we see that for each $s_{i} \in S_{i}, R$ contains a matrix of the form $\alpha_{i}=\operatorname{diag}\left(s_{i} ;{ }^{*}, 0, *\right)$.

Now choose $s_{i} \in S_{i}$ for $i=2,3, \cdots, m$ and let $\alpha_{i}$ be as above. Then $\alpha=\alpha_{2} \alpha_{3} \cdots \alpha_{m} \in R$ and

$$
\alpha=\operatorname{diag}\left(s_{2} s_{3} \cdots s_{m}, 0,0, \cdots, 0\right)=s_{2} s_{3} \cdots s_{m} e_{11}
$$

where $\left\{e_{j k}\right\}$ is the usual set of matrix units. This clearly implies that $R \supseteq S e_{11}$.

Finally let $e_{j k}$ be any matrix unit. Then, by Lemma 1.1(ii), $R \supseteq$ $\rho\left(x_{j}\right)\left(S e_{11}\right) \rho\left(x_{k}^{-1}\right)=S e_{j k}$ and $R \supseteq(S)_{m}$.

Proposition 1.5. Let $K[G]$ satisfy a polynomial identity of degree $n$ and suppose further that $[G: \Delta]<\infty$ and $\Delta$ is abelian. Then $[G: \Delta] \leqq n / 2$.

Proof. By Lemma 5.3 of $[1], K[G]$ satisfies an identity of the form

$$
f\left(\zeta_{1}, \zeta_{2}, \cdots, \zeta_{n}\right)=\zeta_{1} \zeta_{2} \cdots \zeta_{n}+\sum_{\sigma \in \mathrm{S}} k_{\mathrm{ym}_{n} ; \sigma \neq 1} \zeta_{\sigma(1)} \zeta_{\sigma(2)} \cdots \zeta_{\sigma(n)} .
$$

Then of course $\rho(K[G])$ also satisfies $f$. Since $f$ is multilinear and $K[\Delta]$ is central in $K[\Delta]_{m}$, it then follows easily that $R=K[\Delta] \cdot \rho(K[G])$ satisfies $f$. By Lemma $1.4, R \supseteq(S)_{m}$, so $(S)_{m}$ also satisfies $f$.

Suppose by way of contradiction that $m=[G: \Delta]>n / 2$. Since $S$ is not nilpotent by Lemma 1.3 we can choose $s^{(1)}, s^{(2)}, \cdots, s^{(n)} \in S$ with $s^{(1)} s^{(2)} \cdots s^{(n)} \neq 0$. Since $n<2 m$ we may set $\zeta_{1}=s^{(1)} e_{11}, \zeta_{2}=s^{(2)} e_{12}$, $\zeta_{3}=s^{(3)} e_{22}, \zeta_{4}=s^{(4)} e_{23}, \zeta_{5}=s^{(5)} e_{33}, \cdots$. Then $\zeta_{1} \zeta_{2} \cdots \zeta_{n}$ evaluated at these values is $s^{(1)} s^{(2)} \cdots s^{(n)} e_{1 j} \neq 0$ where $j=[n / 2]+1$. On the other hand for all $\sigma \in \operatorname{Sym}_{n}, \sigma \neq 1, \zeta_{\sigma(1)} \zeta_{\sigma(2)} \cdots \zeta_{\sigma(n)}$ evaluated at these values is zero. Thus $(S)_{m}$ does not satisfy $f$, a contradiction. Therefore $m \leqq n / 2$ and the result follows.

2. The general case. Let $\Delta_{k}(G)$ be defined as in [3].

LEMMA 2.1. Suppose there exists an integer $k$ with $\left[G: \Delta_{k}(G)\right]<\infty$. Then $[G: \Delta]<\infty$ and $\left|\Delta^{\prime}\right|<\infty$.

Proof. Since $\Delta \supseteq \Delta_{k}$ and $\left[G: \Delta_{k}\right]<\infty$ we have $[G: \Delta]<\infty$. Now $\Delta$ is a subgroup of $G$ so every right translate of $\Delta_{k}$ in $G$ is either entirely contained in $\Delta$ or is disjoint from it. This implies that $\left[\Delta: \Delta_{k}\right]<\infty$ and say $\Delta=\Delta_{k} y_{1} \cup \Delta_{k} y_{2} \cup \cdots \cup \Delta_{k} y_{r}$. 
Since each $y_{i} \in \Delta$ we can set $u=\max _{i}\left[G: C\left(y_{i}\right)\right]<\infty$. If $x \in \Delta$ then $x \in \Delta_{k} y_{i}$ for some $i$ and this implies easily that $[G: C(x)] \leqq u k$. Thus $\left[\Delta: C_{\Delta}(x)\right] \leqq u k$ and by Theorem 4.4(ii) of $[3],\left|\Delta^{\prime}\right|<\infty$.

We now come to the main result of this paper.

THEOREM 2.2. Let $K[G]$ satisfy a polynomial identity of degree $n$. Then $[G: \Delta(G)] \leqq n / 2$ and $\left|\Delta(G)^{\prime}\right|<\infty$.

Proof. Set $k=(n !)^{2}$. Then by Theorem 3.4 of [3], $\left[G: \Delta_{k}(G)\right]<\infty$. Thus, by Lemma 2.1, $[G: \Delta(G)]<\infty$ and $\left|\Delta(G)^{\prime}\right|<\infty$. Set $H=\Delta(G)^{\prime}$ and consider $\bar{G}=G / H$. If $x \in \Delta(G)$ then clearly $\bar{x}$, its image in $\bar{G}$, has only finitely many conjugates and $\bar{x} \in \Delta(\bar{G})$. Conversely suppose $\bar{x} \in \Delta(\bar{G})$. Then conjugates of $x$ are contained in only finitely many cosets of $H$. Since $H$ is finite, $x$ has only finitely many conjugates and $x \in \Delta(G)$. Thus $\Delta(\bar{G})=\Delta(G) / H$.

Consider $K[\bar{G}]$. Since $K[\bar{G}]$ is an epimorphic image of $K[G]$ we see that $K[\bar{G}]$ satisfies a polynomial identity of degree $n$. Since $\Delta(\bar{G})=\Delta(G) / H$ and $H=\Delta(G)^{\prime}$ we see that $\Delta(\bar{G})$ is abelian and $[\bar{G}: \Delta(\bar{G})]<\infty$. By Proposition 1.5 we have finally $[G: \Delta(G)]=[\bar{G}: \Delta(\bar{G})] \leqq n / 2$ and the result follows.

The following corollary shows that the above bound $n / 2$ is best possible. The result is an immediate consequence of Theorems 1.1(i) and 1.3(i) of [3] and Theorem 2.2.

COROLlary 2.3. Let $n$ be a positive integer and suppose that $G$ is a group with $\Delta(G)$ abelian. Then $[G: \Delta(G)] \leqq n / 2$ if and only if $K[G]$ satisfies a polynomial identity of degree $\leqq n$.

On the other hand, there is no fixed bound for the size of $\Delta(G)^{\prime}$. For example, let $A$ be a finite abelian group of odd order and let $G$ be the extension of $A$ by an element $x$ of order 2 which acts in a dihedral manner on $A$ (that is, $a^{x}=a^{-1}$ for all $a \in A$ ). Then $G$ is finite so $G=\Delta(G)$ and $A=G^{\prime}$ can be made arbitrarily large. Since $G$ has an abelian subgroup of index $2, K[G]$ satisfies a polynomial identity of degree 4 and this is independent of the size of $A=G^{\prime}$.

Finally we remark that Theorem 2.2 answers in the affirmative Problem $4(i)$ of $[2]$.

\section{REFERENCES}

1. D. S. Passman, Linear identities in group rings, Pacific J. Math. 36 (1971), 457-484.

2. - Infinite group rings, Marcel Dekker, New York, 1971.

3. _- Group rings satisfying a polynomial identity, J. Algebra (to appear).

4. M. Smith, On group algebras, Bull. Amer. Math. Soc. 76 (1970), 780-782.

Department of Mathematics, University of Wisconsin, Madison, Wisconsin 53706 